\title{
DETECÇÃO DE INIBIDORES DE PROTEASES EM SEMENTES DE Punica granatum
}

\author{
Lara Franca Colares ${ }^{\mathrm{a}, *}$,Antero Ricardo Santos Neto ${ }^{\mathrm{a}}$, Isac Henrique Cordeiro ${ }^{\mathrm{a}}$, Sandra Bertelli Ribeiro de Castro ${ }^{\mathrm{b}}$, Rodrigo \\ Moreira Verlyc, Caio Cesar de Souza Alves ${ }^{\text {e }}$ Alessandra de Paula Carli ${ }^{a}$ \\ anstituto de Ciências, Engenharia e Tecnologia, Universidade Federal dos Vales do Jequitinhonha e Mucuri, 39803-371 Teófilo \\ Otoni - MG, Brasil \\ 'Departamento de Farmácia, Universidade Federal de Juiz de Fora, 35010-177 Governador Valadares - MG, Brasil \\ 'Departamento de Química, Universidade Federal dos Vales do Jequitinhonha e Mucuri, 39100-000 Diamantina - MG, Brasil \\ 'Faculdade de Medicina do Mucuri, Universidade Federal dos Vales do Jequitinhonha e Mucuri, 39803-371 Teófilo Otoni - MG, Brasil
}

Recebido em 25/07/2016; aceito em 31/10/2016; publicado na web em 21/11/2016

\begin{abstract}
DETECTION OF PROTEASE INHIBITORS IN SEEDS OF Punica granatum. The use of plants as a way to prevent and treat disease comes from ancient times. With the increasing return on consumption of plants for medicinal purposes, the plant-based medicines have gained greater appreciation. Protease inhibitors are compounds that can decrease activity of an enzyme. They are found in plants, especially in Fabaceae, Poaceae, and Solanaceae families, and show satisfactory use to treat diseases due to their regulatory activities and selective proteolytic degradation of target substrates. This study aimed to obtain protease inhibitors in the seeds of Punica granatum, due its use in traditional medicine. The alcohol-acid solution was used to extract and isolate the protease inhibitors in P. granatum seeds and Glycine max grains. The extract of $P$. granatum seeds showed similar concentration of protease inhibitors when compared to G. max. A $14 \mathrm{kDa}$ band was detected by electrophoresis on polyacrylamide gel, which was characterized by high-performance liquid chromatography as protease inhibitors. The extract of $P$. granatum seeds showed a potent trypsin inhibitor activity (EC50 $18.2 \mathrm{ng} \mathrm{mL}^{-1}$ ). This study showed for the first time the extraction, purification, and identification of protease inhibitors in Punica granatum seeds.
\end{abstract}

Keywords: protease inhibitors; Punica granatum seeds; traditional medicine.

\section{INTRODUÇÃO}

As plantas biossintetizam substâncias, conhecidas como metabólitos secundários, que desempenham importantes atividades biológicas na adaptação e na propagação das espécies vegetais. ${ }^{1}$ Assim como os metabólitos secundários, os inibidores de proteases estão presentes em plantas e são encontrados principalmente em leguminosas em geral, das famílias Fabaceae, Poaceae e Solanaceae. ${ }^{2,3}$ Os inibidores de proteases apresentam diferentes funções como: proteína de reserva, agente regulador de proteases endógenas, proteção dos fluídos e tecidos da degradação pela atividade proteolítica, ${ }^{4}$ além da defesa direta ou indireta contra os ataques de insetos e infecções por patógenos. 5

Por terem a capacidade de diminuir a atividade de uma enzima, os inibidores de proteases podem ser enquadrados em três grupos diferentes, levando em consideração suas especificidades: inibidores que reagem com mais de uma classe de proteases, os que são específicos para uma das classes e aqueles que apresentam alta especificidade para uma única protease. ${ }^{6}$ Quando se analisa a estrutura, estes podem ser classificados em grupos que apresentam baixo peso molecular e grupos dos inibidores peptídicos com uma ou mais cadeias polipeptídicas. Existe ainda a classificação de acordo com o tipo de protease que inibem: serino, aspartato, treonino, cisteíno e metalo-proteases. ${ }^{7}$

As serino-proteases possuem seus inibidores subdivididos em 16 classes, entretanto, destacam-se: os inibidores de tripsina do tipo Kunitz, os inibidores de Bowman-Birk (BBI) e os inibidores presentes na batata do tipo I e II. ${ }^{8}$

Pesquisas têm demonstrado a utilização de inibidores de proteases no tratamento de enfermidades devido suas funções de regulação

*e-mail: laraf_colares@hotmail.com de diferentes processos em que as proteases estão envolvidas, que englobam a quebra de proteínas intracelulares, transcrição, ciclo celular, invasão celular e apoptose, através da degradação seletiva de substratos alvo. ${ }^{4,9}$

A Punica granatum é árvore de pequeno porte oriunda da África boreal, facilmente cultivada nas regiões quentes e temperadas do mundo inteiro, que pertence à família Lythraceae (antiga Punicaceae) e apresenta taninos, alcaloides, amido, ácido gálico, vitaminas B2, C e D, magnésio e ferro em seus constituintes. ${ }^{10}$ Apesar dos inibidores de proteases serem tradicionalmente encontrados em leguminosas, a $P$. granatum foi selecionada para se avaliar a capacidade de obtenção de inibidores de proteases, nunca encontrados nas sementes dessa espécie vegetal, visto a sua utilização na medicina tradicional.

\section{PARTE EXPERIMENTAL}

\section{Purificação de inibidores de proteases em sementes de Punica granatum}

Os frutos, para separação das sementes de $P$. granatum, foram coletados no município de Araçuaí-MG em outubro e novembro de 2015. O grão de Glycine max foi adquirido no comércio local de Teófilo Otoni-MG. A extração proteica na G. max foi realizada como medida padrão de comparação para inibidores de proteases, seguindo a metodologia proposta por Yavelow e seus colaboradores, ${ }^{11}$ a qual foi adaptada para as sementes de $P$. granatum.

Para a extração, $100 \mathrm{~g}$ das sementes foram trituradas. Após a trituração foi acrescentado $10 \%$ p/v de etanol a $60 \%$ e mantido por $1 \mathrm{~h}$ a $55^{\circ} \mathrm{C}$ com agitação constante e, em seguida, foi resfriado rapidamente em banho de gelo e disposto em repouso por 24 horas a $4{ }^{\circ} \mathrm{C}$. $\mathrm{O}$ material oriundo desse tratamento foi submetido à centrifugação 
a $6.500 \times g$, por 30 min a $4{ }^{\circ} \mathrm{C}$ para remoção de fibras insolúveis presentes. A mistura foi então filtrada em gaze e acidificada com $\mathrm{HCl}$ até atingir $\mathrm{pH}$ 5,3. Procedeu-se a precipitação dos inibidores de proteases, com a adição de volumes crescentes de Acetona P.A. $\left(-20^{\circ} \mathrm{C}\right)$, mantido em banho de gelo. $\mathrm{O}$ extrato enriquecido obtido foi submetido à centrifugação a $6.500 \times g$ por $30 \mathrm{~min}$. O precipitado foi suspenso em $5 \mathrm{~mL}$ de água destilada e armazenado a $-80{ }^{\circ} \mathrm{C}$ para posterior análise.

\section{Espectrofotometria do extrato enriquecido de inibidores de proteases}

A concentração de proteínas foi determinada pelo método de Bradford ${ }^{12} \mathrm{e}$ a análise foi realizada no espectrofotômetro AJX-6100PC (Espectrofotômetro Duplo Feixe, MICRONAL) no comprimento de onda 595 nanômetros $(\mathrm{nm})$. Os dados foram gerados pelo software UV-Vis Analyst versão 5.3. Após este procedimento, os extratos enriquecidos de sementes de $P$. granatum e grãos de $G$. max foram diluídos em água destilada (100x) e submetidos à varredura de comprimento de onda entre 199 e $700 \mathrm{~nm}$ (UV-Vis Cary 50, Varian). Os dados foram gerados pelo software Scan versão 3.0 e analisados pelo programa OriginPro versão 8.0.

\section{Eletroforese em gel de poliacrilamida e SDS: Detecção dos inibidores de proteases}

As amostras provenientes da purificação foram submetidas à detecção por eletroforese em gel de poliacrilamida a $12 \%$ SDS-PAGE. Foi usado o padrão de peso molecular de 10 a $250 \mathrm{kDa}$ (PageRuler Plus Prestained Protein Ladder, Thermo Scientific). A coloração foi realizada com Coomassie Blue R-250 a 0,025\% em metanol $25 \%$ e $5 \%$ de ácido acético, em agitação branda de 2 horas. Em seguida os géis foram descorados em solução de $40 \%$ metanol e $10 \%$ ácido acético até a visualização plena das bandas.

\section{Cromatografia líquida de alta eficiência dos extratos} enriquecidos de inibidores de proteases

As amostras dos sobrenadantes dos extratos enriquecidos de $P$. granatum e G. max foram diluídas em água destilada (1:10). $20 \mu \mathrm{L}$ desta diluição foram submetidos à cromatografia líquida de alta eficiência em coluna $\mathrm{LC}_{18}(25 \mathrm{~cm}$ x 4,6 mm) (Supelcosi, Sigma-Aldrich) em sistema de HPLC (ProStar, Varian) sob um fluxo de $0,8 \mathrm{~mL} \mathrm{~min}^{-1}$ durante 45 minutos e os dados analisados no programa Origin.

\section{Potencial Zeta do extrato enriquecido de inibidores de proteases}

As amostras para determinação do potencial Zeta dos inibidores de proteases foram preparadas a partir da diluição de 10 vezes do sobrenadante do extrato enriquecido da $P$. granatum em água destilada, e medida por meio de espalhamento de luz dinâmico (DLS) (Zetasizer Nano - ZS, Malvern) no software Zetasizer versão 7.11.

\section{Atividade inibitória anti-tripsina}

O substrato utilizado foi o cloridrato de N- $\alpha$-benzoil-DLarginil-p-nitroanilida (DL-BApNA), preparado dissolvendo 196 mg do composto em $5 \mathrm{~mL}$ de DMSO (dimetilsulfóxido) para uma concentração final de $9 \times 10^{-2} \mathrm{~mol} \mathrm{~L}^{-1}$. Esta solução foi mantida no congelador como solução estoque e diluída no momento do uso com Tris- $\mathrm{HCl} 0,1$ mol L-1 contendo $\mathrm{MgCl}_{2}, 20$ mmol L-1 e $\mathrm{pH} 8,1$ para a concentração de $9 \times 10^{-4} \mathrm{~mol} \mathrm{~L}^{-1}$.
Para obter uma concentração que inibe entre $50 \%$ da atividade da tripsina, foram realizadas diluições seriadas do inibidor. A cada tubo de ensaio se adicionou-se $200 \mu \mathrm{L}$ de DL-BApNA, $20 \mu \mathrm{L}$ de tripsina $\left(0,05 \mathrm{mg} \mathrm{mL}^{-1}\right)$ e $20 \mu \mathrm{L}$ do inibidor. Nos tubos brancos foram adicionados $200 \mu \mathrm{L}$ de substrato e $40 \mu \mathrm{L}$ de água, enquanto que os tubos controles de atividade enzimática continuaram com $200 \mu \mathrm{L}$ de substrato, $20 \mu \mathrm{L}$ de enzima e $20 \mu \mathrm{L}$ de água.

Os tubos de reação passaram por agitação em vórtex e foram posteriormente incubados em banho-maria a $37^{\circ} \mathrm{C}$ por aproximadamente 40 minutos. A reação foi interrompida com $40 \mu \mathrm{L}$ de ácido acético $60 \% \mathrm{v} / \mathrm{v}$ e adicionado $250 \mu \mathrm{L}$ de água destilada. A quantificação da atividade inibitória ocorreu em espectrofotômetro AJX-6100PC (Espectrofotômetro Duplo Feixe, MICRONAL) no comprimento de onda 410 nanômetros $(\mathrm{nm})$. Os dados foram gerados pelo software UV-Vis Analyst versão 5.3.

A concentração do extrato enriquecido a base de sementes de $P$. granatum que induz metade do efeito máximo (EC50) foi calculada através da análise da regressão não-linear da curva.

\section{RESULTADOS E DISCUSSÃO}

\section{Punica granatum apresenta quantidade de proteínas semelhante à Glycine max}

O extrato enriquecido a base de sementes de $P$. granatum teve uma concentração de proteínas semelhante à obtida na G. max grão (Figura 1).

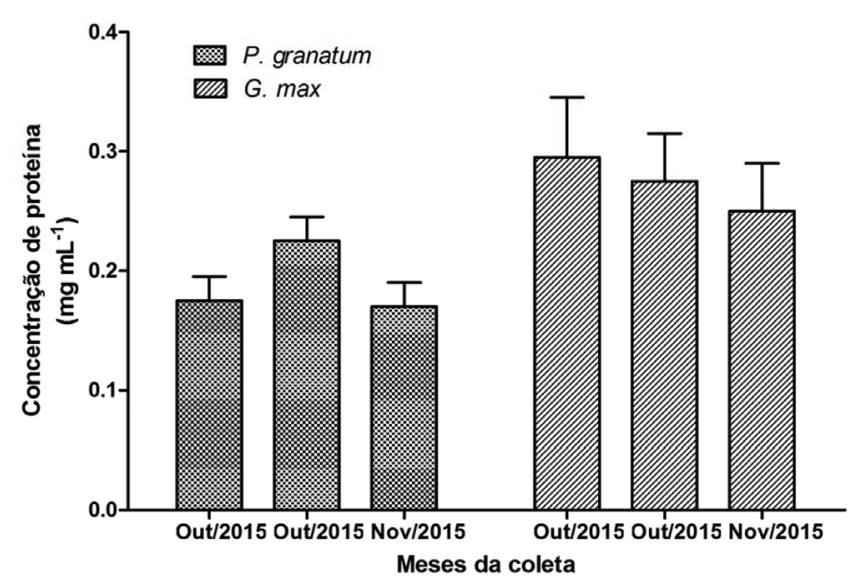

Figura 1. Quantificação de inibidores de proteases do extrato enriquecido de sementes de Punica granatum e de Glycine max. Os extratos enriquecidos de sementes de P. granatum e a base de G. max grão, obtidos de extrações em diferentes datas foram detectadas pelo método de Bradford (1976) a uma absorbância de $595 \mathrm{~nm}$. Barras=média \pm desv.padrão

A metodologia para extração proteica proposta por Yavelow e seus colaboradores ${ }^{11}$ foi empregada pela primeira vez em sementes de $P$. granatum para isolamento e purificação de inibidores de proteases.

\section{Intensidade de absorbância para inibidores de proteases em} Punica granatum

O espectro de absorção mostrou para cada espécie dois picos para inibidores de proteases. O extrato enriquecido de $P$. granatum teve seus picos em $212 \mathrm{~nm}$ e $268 \mathrm{~nm}$ e o extrato de G. max apresenta seus picos em $235 \mathrm{~nm}$ e $268 \mathrm{~nm}$ (Figura 2).

Os picos identificados na $P$. granatum podem ser caracterizados como inibidores de proteases de acordo com o demonstrado por Leal ${ }^{13}$ e Clemente e colaboradores ${ }^{14}$ em seus estudos com extratos de $G$. 


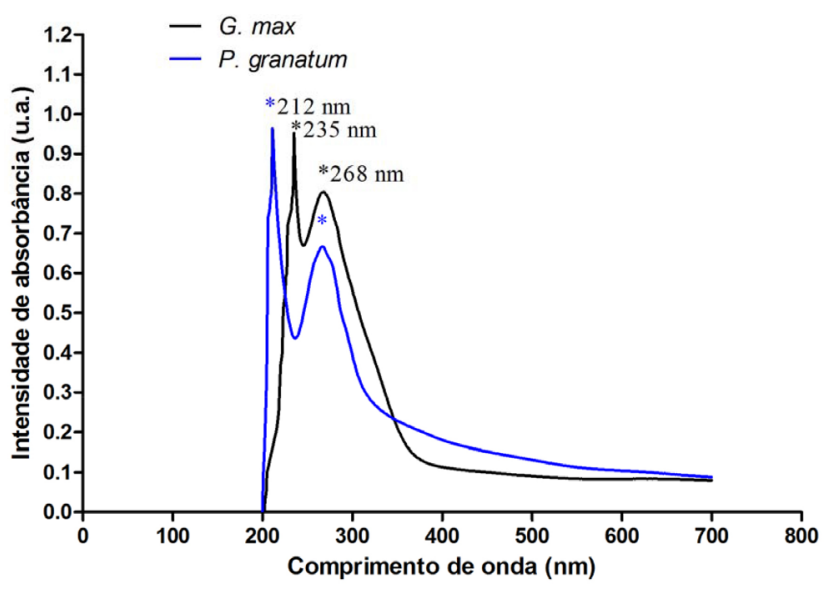

Figura 2. Espectro de absorção molecular de inibidores de proteases dos extratos enriquecidos a base de sementes de Punica granatum (- ) e grãos de Glycine max ( - ). As amostras apresentaram curvas analíticas de absorção em $212 \mathrm{~nm}$ para P. granatum, $235 \mathrm{~nm}$ para G. max e $268 \mathrm{~nm}$ para ambos os extratos, sendo monitorados de 199 a $700 \mathrm{~nm}$. A varredura foi realizada com as amostras diluídas em água destilada e a temperatura ambiente

max, que observaram picos de intensidade de absorbância.

Os valores diferentes podem ser referentes à absorção dos dois sítios ativos dos inibidores de proteases, podendo ser a absorção em $268 \mathrm{~nm}$ referente à quimotripsina que, como demonstrado por estudos de Apura e colaboradores, ${ }^{15}$ está bem próxima da absorbância em UV de $250 \mathrm{~nm}$ da $\alpha$-quimotripsina, isso devido sua constituição por vários aminoácidos aromáticos, e, quanto à absorção em 212 nm para o extrato de semente de $P$. granatum, a mesma se refere à absorbância da tripsina.

\section{Detecção de inibidores de proteases em Punica granatum por eletroforese}

O gel de poliacrilamida identificou a presença de uma banda de inibidor de proteases na altura de $14 \mathrm{kDa}$ (Figura 3). Uma banda com o mesmo peso molecular foi encontrada no extrato a base de Glycine max grão, utilizado como padrão de comparação. Em $G$. max também foram detectadas bandas nas alturas de 17, 21 e $30 \mathrm{kDa}$ aproximadamente.

Estudos com a leguminosa da espécie Vigna sp. têm demonstrado o peso molecular dos inibidores de proteases de tripsina e do tipo Bowman-Birk (BBI) como sendo de $10-16 \mathrm{kDa},{ }^{16,17}$ podendo essa banda representar a forma do dímero do inibidor, comportamento recorrente em inibidores $\mathrm{BBI}$ encontrado em várias leguminosas. ${ }^{18}$ Desta forma, no presente trabalho, a banda de $14 \mathrm{kDa}$ pode ser considerada como sendo um dímero de inibidor de quimotripsina e tripsina.

A presença de diversas massas moleculares proteicas na preparação do extrato a base de G. max grão é justificável pelo fato da mesma possuir ampla distribuição de inibidores de proteases do tipo Kunitz e lectinas em suas sementes, que apresentam massas moleculares em torno de 21-22 e 30-35 kDa, ${ }^{19}$ respectivamente, sugerindo, assim, que as demais bandas aparentes no perfil eletroforético sejam das mesmas.

Diante disso, o extrato enriquecido de sementes de $P$. granatum demonstrou por meio do perfil eletroforético a presença de inibidor de proteases detectado pela absorbância apresentada no espectro e absorção (Figura 2).

A intensidade da curva analítica de absorção do extrato de $P$. granatum relativamente semelhante com a da G. max grão pode estar relacionada com a concentração de inibidores de proteases presente nos extratos, como demonstrado por meio das bandas aparentes no gel de poliacrilamida.

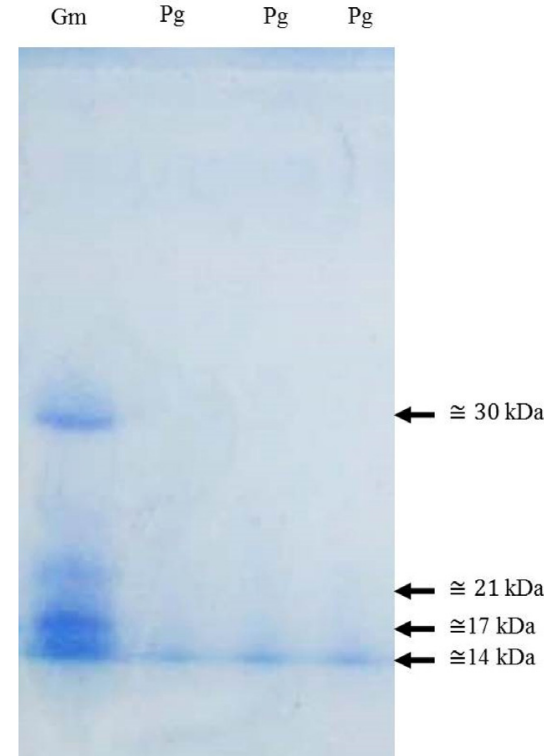

Figura 3. Perfil eletroforético (12\% SDS-PAGE) do extrato enriquecido a base de sementes de Punica granatum. Legenda: Gm: extrato a base de Glycine max grão (padrão de inibidores de proteases). Pg: extrato a base de sementes de P. granatum. Foi aplicado em cada poço, $20 \mu \mathrm{l}$ de amostra. Gel corado com Coomassie Blue $R-250$. Seta=peso molecular aproximado das bandas ( $k D a)$

Perfil cromatográfico igual para a Punica granatum e a Glycine max para presença de inibidores de proteases

O cromatograma detectou para ambos os extratos dois picos simétricos com tempos de retenção em 12 e 13 minutos, e outros dois picos em 28 e 40 minutos com intensidades diferentes (Figura 4).

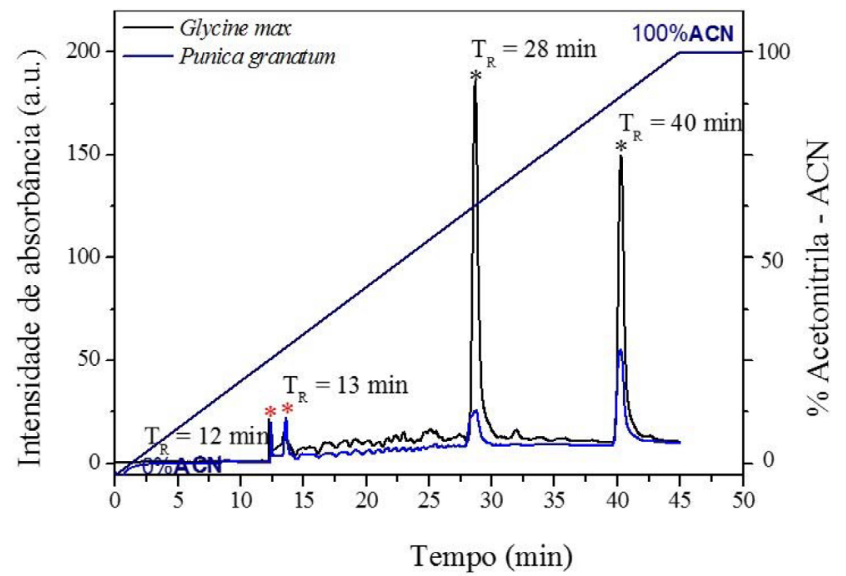

Figura 4. Perfil cromatográfico em HPLC obtido da leitura da amostra do extrato enriquecido a base de sementes de Punica granatum e Glycine max grão. Condições cromatográficas: Coluna utilizada $L C_{18}(25 \mathrm{~cm} \times 4,6 \mathrm{~mm})$ Supelcosil, 2,2 mL. Fase Móvel: $A: \mathrm{H}_{2} \mathrm{O}+\mathrm{TFA}$ 0,1\%; $\mathrm{B}$ : ACN + TFA 0,08\%. Eluição por gradiente: 0 a 100\% B em $45 \mathrm{~min}$; fluxo: 0,8 $\mathrm{mL} \mathrm{min}^{-1}$. *Picos de absorção de inibidores de proteases de interesse

Os dois primeiros picos de retenção (12 e 13 minutos) possivelmente representam na $G$. $\max$ os inibidores de proteases da família Bowman-Birk (BBI), ${ }^{13,20}$ e são os mesmos referentes ao dímero do inibidor de proteases detectados no gel de poliacrilamida em $14 \mathrm{kDa}$. $\mathrm{O}$ fato de aparecerem dois picos no cromatograma referentes a apenas uma banda de inibidor de proteases no gel, pode estar relacionado com a não divisão do dímero em duas isoformas, comportamento recorrente para inibidores de proteases. ${ }^{18}$ 
O cromatograma apresentou, ainda, outros dois picos em tempos de retenção de 28 e 40 minutos, que supostamente representam alguma proteína que não foi detectada no extrato de P. granatum pelo gel de poliacrilamida, devido a sua baixa sensibilidade comparada ao sistema de HPLC para detecção de partículas, ou possível falha no processo de coloração do gel.

Já para o extrato de G. max os picos nos tempos de retenção de 28 e 40 minutos tiveram uma maior intensidade e simetria, podendo ser a representação das outras bandas aparentes no gel, uma vez que a G. max possui ampla distribuição de inibidores de proteases do tipo Kunitz e das lectinas em suas sementes, que apresentam massas moleculares em torno de 21-22 e 30-35 kDa, respectivamente. ${ }^{19}$

Sendo assim, tendo o perfil cromatográfico do extrato de grãos de G. max como padrão de comparação, pode-se supor que os tempos de retenção de 12 e 13 minutos são referentes à presença de inibidores de proteases do tipo BBI. Os demais picos em 20 e 48 minutos, assim como descrito para as leguminosas, nos extratos de $P$. granatum e G. $\max$, possivelmente são relativos aos inibidores de proteases do tipo Kunitz e lectina.

No que se refere à afinidade com a coluna, os dois primeiros picos de ambos os extratos enriquecidos tiveram uma hidrofobicidade de 40 e 43,3\%, referentes aos tempos de retenção de 12 e 13 minutos respectivamente, o que pode confirmar que essas proteínas detectadas são relativamente hidrofílicas e detentoras de baixo peso molecular, característica de inibidores de proteases do tipo BBI. ${ }^{9}$

\section{Distribuição do potencial Zeta dos inibidores de proteases}

Conforme demonstrado na Tabela 1, a amostra do extrato de Punica granatum obteve um valor negativo como o esperado para proteínas.

Tabela 1. Potencial Zeta e das partículas dos inibidores de proteases

\begin{tabular}{lc}
\hline & Potencial Zeta $\zeta(\mathrm{mV})$ \\
\hline Punica granatum & $-22,9$ \\
\hline
\end{tabular}

Considerando que a concentração de proteínas exerce um aumento no valor do potencial Zeta, indicando assim a formação de partículas com maior densidade de carga superficial, ${ }^{21} \mathrm{o}$ extrato de sementes de $P$. granatum mostrou uma concentração significativa de inibidores de proteases.

\section{Atividade anti-tripsina}

Para a caracterização dos inibidores obtidos no gel de poliacrilamida e no HPLC foi realizada a atividade anti-tripsina do extrato enriquecido a base de sementes de $P$. granatum. O extrato apresentou uma potente ação inibitória da atividade da tripsina (Figura 5). A inibição de $50 \%$ da atividade da tripsina (EC50) foi alcançada na concentração de $1,82 \times 10^{-5} \mathrm{mg} \mathrm{mL}^{-1}$.

$\mathrm{O}$ inibidor do tipo BBI, que apresenta banda característica entre 10 e $17 \mathrm{kDa}$ e com picos de tempo de retenção aos 12 e 13 minutos no HPLC, é descrito como um potente redutor da atividade da tripsina, sugerindo que o produto obtido no extrato enriquecido das sementes de $P$. granatum seja do tipo BBI. Novos estudos devem ser realizados para a caracterização dos picos com tempo de retenção em 28 e 40 min encontrados no HPLC.

\section{CONCLUSÃO}

O presente estudo demonstrou pela primeira vez a detecção e purificação de inibidores de proteases em sementes de Punica granatum.

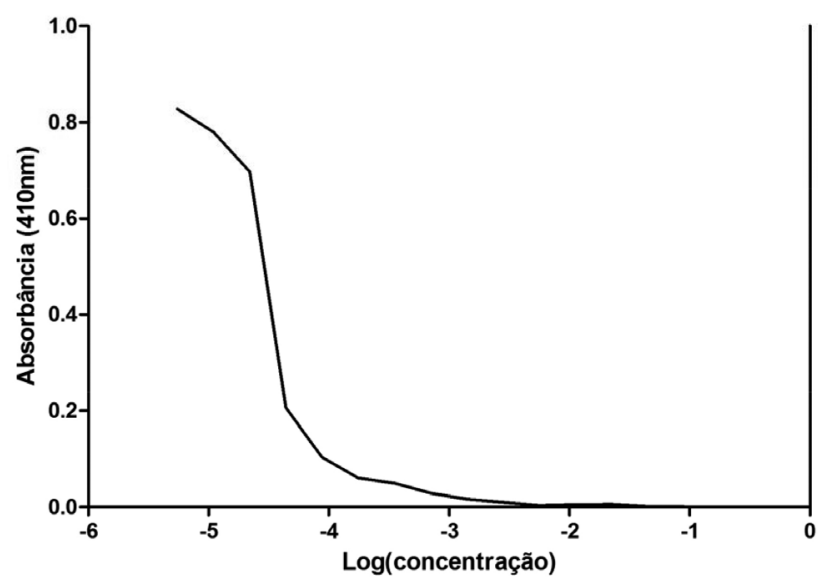

Figura 5. Atividade anti-tripsina do extrato enriquecido a base de sementes de Punica granatum. $20 \mu \mathrm{L}$ de diluições seriadas do inibidor foram adicionadas a cada tubo de ensaio que continha $200 \mu L$ de DL-BApNA ( $N$ - $\alpha$-benzoil-DLarginil-p-nitroanilida) e $20 \mu \mathrm{L}$ de tripsina $\left(0,05 \mathrm{mg} \mathrm{mL}^{-1}\right)$. A quantificação da atividade inibitória foi analisada em $410 \mathrm{~nm}$. A EC50 foi calculada através da análise da regressão não-linear da curva

A caracterização do inibidor de protease da família Bowman-Birk em sementes de Punica granatum foi comprovada por meio do gel de poliacrilamida com a detecção da presença de bandas, dos perfis cromatográficos em HPLC e da atividade enzimática característicos deste inibidor.

\section{AGRADECIMENTOS}

Os autores agradecem ao LASEB - Laboratório de Síntese e Estrutura de Biomoléculas da Universidade Federal dos Vales do Jequitinhonha e Mucuri - UFVJM / Campus JK e ao PPGTAS Programa de Pós-Graduação em Tecnologia, Ambiente e Sociedade da UFVJM / Campus Mucuri pelo apoio ao desenvolvimento desse estudo.

\section{REFERÊNCIAS}

1. Dewick, P. M.; Medicinal Natural Products - A Biosynthetic Approach, $2^{\text {a }}$ ed., John Wiley \& Sons: England, 2002.

2. Hammond, R. W.; Foard, D. E.; Larkins, B. A.; J. Biol. Chem. 1984, 259, 9883.

3. Baek, J. M.; Song, J. C.; Choi, Y. D.; Kim, S. I.; Biosci., Biotechnol., Biochem. 1994, 58, 843.

4. Ryan, C. A.; Annu. Rev. Phytopathol. 1990, 28, 425; Richardson, M.; Methods Plant Biochem. 1991, 5, 259; Walker, A. J.; Ford, L.; Majerus, M. E. N.; Geoghegan, A. E.; Birch, N.; Gatehouse, J. A.; Gatehouse, A. M. R.; Insect Biochem. Mol. Biol. 1997, 28, 173.

5. Carlini, C. R.; Grossi-de-Sa, M. F.; Toxicon 2002, 40, 1515; Lawrence. P. K.; Koundal. K. R.; Electron. J. Biotechnol. 2002, 5, 93.

6. Tremacoldi, C. R.; Proteases e inibidores de proteases na defesa de plantas contra pragas, $1^{\mathrm{a}}$ ed. Versão Eletrônica, Embrapa Amazônia Oriental: Belém, 2009.

7. Power, J. C.; Asgian, J. L.; Ekici, Õ. C.; James, K. E.; Chem. Rev. 2002, $102,4639$.

8. Ryan, C. A.; Annu. Rev. Phytopathol. 1990, 28, 425.

9. Touil, T.; Ciric, B; Ventur, E; Shindler, K.; S.; Gran, B.; Rostami, A.; J. Neurol. Sci. 2008, 271, 191; Clardy, J.; Walsh, C.; Nature 2004, 432, 829.

10. Grandi, T. S. M.; Tratado das plantas medicinais: mineiras, nativas e cultivadas, $1^{\text {a }}$ ed., Adaequatio Estúdio: Belo Horizonte, 2014.

11. Yavelow, J.; Collins, M.; Birk, Y.; Troll, W.; Kennedy, A. R.; Proc. Natl. Acad. Sci. U. S. A. 1985, 82, 5395. 
12. Bradford, M.; Anal. Biochem. 1976, 72, 248.

13. Leal, S. C.; Dissertação de Mestrado, Universidade Federal de Ouro Preto, Brasil, 2010.

14. Clemente, A.; Moreno, F. M.; Manzono, M. Del C. M.; Jimenez, E.; Domoney, C.; Mol. Nutr. Food Res. 2010, 54, 396.

15. Apura, J.; Mendes, M.; Uva, M.; Instituto Superior Técnico 2012, 14.

16. Kumar, K.; Kansal, R.; Subrahmanyam, B.; Koundal, K. R.; Miglani, K.; Gupta, V. K.; Acta Physiol. Plant. 2013, 35, 1887.

17. Sammour, R. H. A.; Turk. J. Biol. 2006, 30, 207.
18. Bergeron, D.; Nielsen, S. S.; J. Agric. Food Chem. 1993, 41, 1544; Fields, C.; Mallee, P.; Muzard, J.; Lee, G. U.; Food Chem. 2012, 134, 1831.

19. Song, H. K.; Suh, S. W.; J. Mol. Biol. 1998, 275, 347; Sharon, N.; Lis, H.; FASEB J. 1990, 4, 3198.

20. Gariani, T.; Leatherbarrow, R. J.; J. Pept. Res. 1997, 49, 467.

21. Fayad, S. J.; Ramos, B. G. Z.; Soldi, V.; Minatti, E.; Quim. Nova 2015, $38,91$. 